\title{
Corrigendum: 'Generalized multiple-prism dispersion theory for pulse compression in ultrafast dye lasers'
}

\section{F. J. DUARTE, Optical and Quantum Electronics 19 (1987) 223.}

It has been found that the numerical values of $(d n / d \lambda)$ and $\left(d^{2} n / d \lambda^{2}\right)$, for ZnSe, are in error. Using the equation for $n(\lambda)$ given by Marple [1] the respective values, at $\lambda=0.62 \mu \mathrm{m}_{*}$ are estimated to be $(\mathrm{d} n / \mathrm{d} \lambda)=-0.698 \mu \mathrm{m}^{-1}$ and $\left(\mathrm{d}^{2} n / \mathrm{d} \lambda^{2}\right)=5.068 \mu \mathrm{m}^{-2}$.

The revised $d n / d \lambda)$ and $\left(d^{2} n / d \lambda^{2}\right)$ modify the estimated value for the required prism separation length, necessary to achieve negative dispersion in the $\mathrm{ZnSe}$ isosceles prism sequence. The value of $l \geq 11.237 \mathrm{~mm}$ given thus becomes $l \geq 15.499 \mathrm{~mm}$. Also, for the isosceles prism sequence designed to achieve an internal beam expansion factor of 10 with a $150 \mathrm{~mm}$ prism separation $\left(\mathrm{d}^{2} P / \mathrm{d} \lambda^{2}\right)=-8555.329$.

It should be emphasized that the use of the revised values for the $\mathrm{ZnSe}$ dispersion and its derivative does not change in any form the original interpretation that ' $\mathrm{ZnSe}$ can have a substantial effect in augmenting the overall negative dispersion, thus reducing the requirement on prism separation'. Further, this correction does not alter any of the equations, the content of the table, or any of the conclusions of the original paper.

\section{Reference}

1. D. T. F. MARPLE, J. Appl. Phys. 35, (1964) 539. 